\title{
Control of Structural Rhythms in the Lateral Eye of Limulus: Interactions of Natural Lighting and Circadian Efferent Activity
}

\author{
Steven C. Chamberlain and Robert B. Barlow, Jr. \\ Institute for Sensory Research, Syracuse University, Syracuse, New York 13244
}

The structure of the Limulus polyphemus retina changes with the time of day. The structural changes exhibit a circadian rhythm when the animal is kept in constant darkness. During subjective night, the aperture of each visual unit (ommatidium) widens and flattens, allowing more photons to impinge on the underlying photoreceptors. In addition, the photosensitive region (rhabdom) of the photoreceptors widens and shortens. During subjective day, these changes reverse: both the aperture and rhabdom elongate and narrow, reducing the number of photons caught by the photoreceptors.

The endogenous structural rhythm is mediated by efferent optic nerve activity generated from a circadian clock located in the brain. Eliminating the efferent input by cutting the optic nerve blocks the rhythm and shocking the cut nerve restores it.

Natural lighting magnifies the structural rhythm. Daylight induces photomechanical movements, enhancing the endogenous daytime changes that decrease photon catch. The onset of darkness enhances endogenous nighttime changes that increase photon catch. Cutting the optic nerve blocks all structural changes, both endogenous and photomechanical.

In the animal's natural environment, the effects of circadian efferent input and natural lighting are thus reciprocal: The evening onset of efferent activity pushes retinal structure in one direction; the morning onset of daylight pulls it in the other. The 2 processes, however, are not equivalent. In the absence of cyclic lighting, circadian efferent input produces an attenuated structural rhythm, but cyclic lighting alone produces no rhythm.

Circadian rhythms in structure and function are prominent features of the lateral eye of the horseshoe crab Limulus polyphemus. Circadian rhythms in structure include changes in organelles within photoreceptor cells and changes in the position and shape of the photoreceptor and other retinal cells (Behrens, 1974; Chamberlain and Barlow, 1977, 1981; Barlow and Chamberlain, 1980a). Such structural rhythms widen the acceptance angle of individual ommatidia and increase the photon catch by up to 100 times (Barlow et al., 1980). Circadian rhythms in function

\footnotetext{
Received Aug. 29, 1986; revised Dec. 8, 1986; accepted Dec. 22, 1986.

This research was supported by NIH Grants EY-03446 and EY-00667 and NSF Grant BNS 83-20315. We thank William Dossert, Robert Fladd, Andy Vadasz, and Leslie Eisele for technical assistance. We also thank Len Kass, George Renninger, and Bruce Calman for helpful discussions during the progress of this work

Correspondence should be addressed to Dr. Steven C. Chamberlain at the above address.

Copyright (c) 1987 Society for Neuroscience $0270-6474 / 87 / 072135-10 \$ 02.00 / 0$
}

include increases in photoreceptor gain (Renninger et al., 1984) and temporal-frequency response (Batra, 1983) and decreases in photoreceptor noise (Kaplan and Barlow, 1980) and retinal inhibition (Batra and Barlow, 1982). These circadian rhythms combine to increase retinal sensitivity as much as 100,000 -fold at night (Barlow et al., 1985).

The circadian rhythms in the Limulus retina are mediated by the activity of efferent fibers that innervate the retinal cells via the lateral optic nerve (Fahrenbach, 1973, 1981). A clock in the brain generates the efferent activity (Barlow, 1983), which in turn causcs retinal release of the ncurotransmitter octopamine (Battelle et al., 1982; Battelle and Evans, 1984; Kass and Barlow, 1984) and perhaps a second neurotransmitter (Engbretson and Chamberlain, 1981; Lehman and Barlow, 1986). In addition to mediating circadian rhythms in retinal structure and function, the efferent activity also primes the photoreceptors for the daily shedding of photosensitive membrane (Chamberlain and Barlow, 1979, 1984).

The circadian efferent input to the lateral eye does not alone account for the full range of structural rhythms detected under natural lighting. Preliminary studies indicate that the diurnal light cycle has an important role in amplifying the endogenous circadian rhythms (Chamberlain and Barlow, 1981). We report here a detailed study of the rhythms in retinal structure and of the interaction between circadian efferent activity and diurnal lighting that produces them.

\section{Materials and Methods}

Animals. Male and female adult horseshoe crabs (Limulus polyphermus) measuring $20-25 \mathrm{~cm}$ across the carapace were used for this study. For experiments carried out in June at the Marine Biological Laboratory (Woods Holc, MA), freshly collected animals were stored under natural lighting in aquaria with circulated seawater or in pens in the ocean. For experiments performed during other months in our laboratories in Syracuse, animals were maintained in aquaria with recirculated artificial seawater (Instant Ocean, Aquarium Systems, Eastlake, $\mathrm{OH}$ ) in a room illuminated by a skylight. The animals were regularly fed fresh clams.

Fixation and embedding. Whole retinas were fixed without a change in ambient illumination by subretinally injecting a fixative containing $4.5 \%$ sucrose, $3 \% \mathrm{NaCl}, 0.8 \%$ glutaraldehyde, and $5 \%$ formaldehyde in $0.1 \mathrm{M}$ Sorensen's phosphate buffer, $\mathrm{pH}$ 7.2. After remaining $30 \mathrm{~min}$ in the appropriate light or dark condition, the retina and cornea were dissected free and fixed overnight by immersion in fixative at $4^{\circ} \mathrm{C}$. They were then washed in $8 \%$ sucrose in phosphate buffer, cut into small blocks, and postfixed in $1 \% \mathrm{OsO}_{4}$ in phosphate buffer for $1 \mathrm{hr}$ at room temperaturc. Finally they werc washed in distilled water, dehydrated through an ethanol series to propylene oxide, and embedded in EponAraldite.

Light microscopy. For observation with the light microscope and morphometric analysis, serial $1 \mu \mathrm{m}$ sections were cut with a Sorvall MT2-B ultramicrotome (Dupont-Sorvall, Newtown, CT) using glass knives. Each plastic block contained about 40 ommatidia. Sections were oriented perpendicular to the optic axes of ommatidia in the center of 

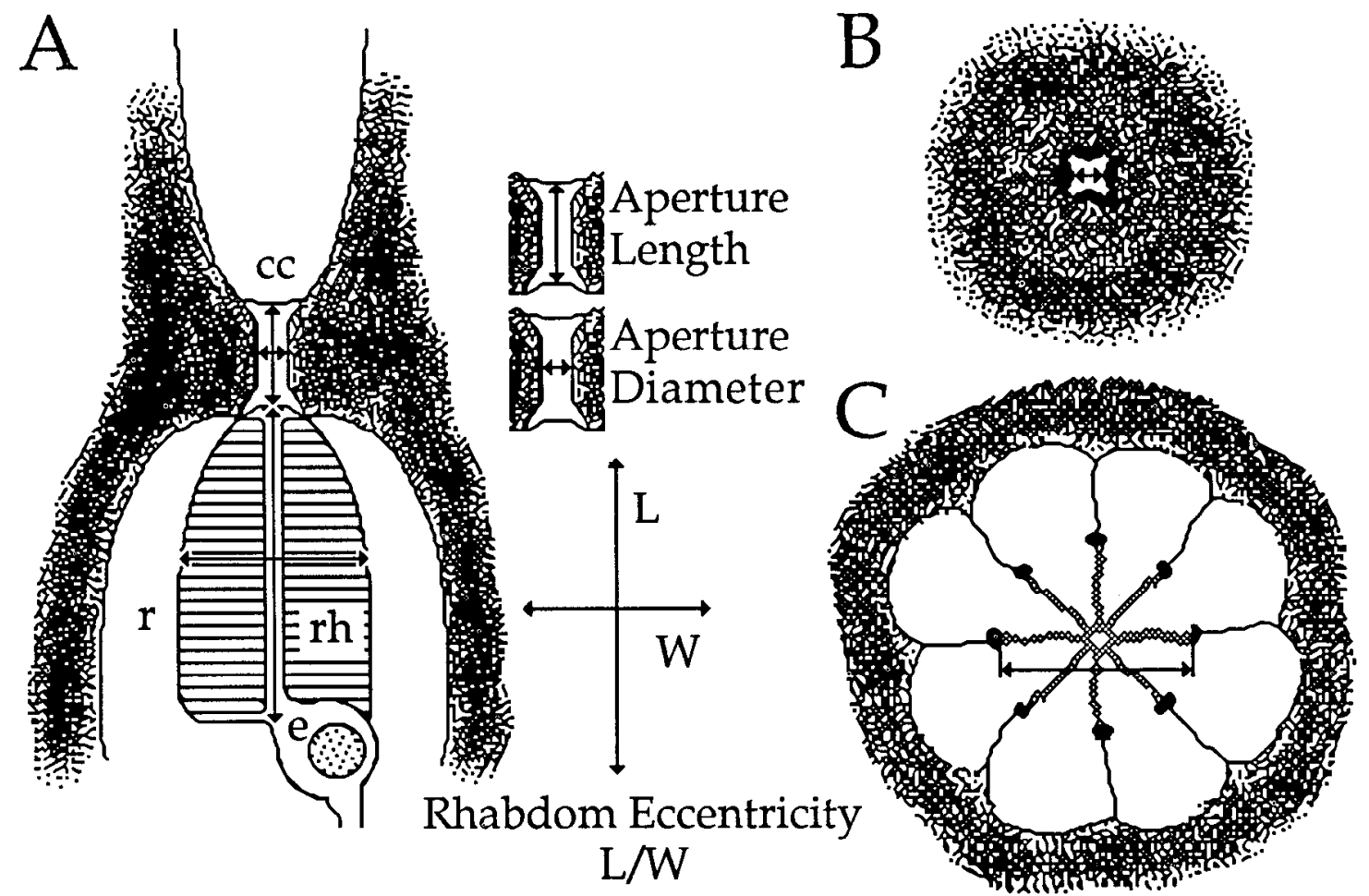

Figure 1. Diagrammatic anatomy of the ommatidia of the Limulus lateral eye showing the parameters measured in this study (arrows). A, Longitudinal section through the middle of an ommatidium. This plane contains all the measured parameters, aperture length, aperture diameter, rhabdom length, and rhabdom width. $c c$, Cuticular cone; $e$, eccentric cell; $r$, retinular cell; $r h$, rhabdom. $B$, Typical narrow aperture as it might appear in the section perpendicular to the plane of $A$ at the midpoint of the aperture length. The minimum aperture diameter is measured in this section (arrow). $C$, Typical section perpendicular to the plane of $A$ at the midpoint of the rhabdom length. The distance from the end of one ray through the eccentric cell dendrite to the end of the other ray is measured along the minor axis of the clliptical profile (arrow). The pigment within the retinular cells is not shown.

each block. Sections were mounted on glass slides and stained with $1 \%$ toluidine blue (Trump et al., 1961). The location of sections on each slide was mapped and serial sections were numbered. One or more sections were traced with a drawing tube and 20 ommatidia were chosen for measuring and numbered on the section map.

Morphometric measurements. Quantitative measurements were made of the following 4 parameters (Fig. 1): the length of the aperture along the optic axis between the base of the cuticular cone and the distal tip of the rhabdom; the length of the rhabdom along the optic axis; the width of the aperture at the midpoint of its length; and the width of the rhabdom at the midpoint of its length. For each ommatidium studied, we determined the number of sections containing (A) the tip of the cuticular cone over the center of the ommatidium; (B) the peripheral tip of the eccentric cell; and (C) the most proximal portion of rhabdom. $A-B$ was taken to be the length of the aperture along the optic axis, and $\mathrm{B}-\mathrm{C}$ was taken to be the length of the rhabdom. We calculated the section number, $(A-B) / 2$, of the point midway along the length of the aperture and measured the minimum diameter of the aperture in that section. We then calculated the section number, $(B-C) / 2$, of the point midway along the length of the rhabdom and measured the minimum diameter of the rhabdom in that section. To measure the shape of the rhabdom, we determined the ratio of rhabdom length to width. This ratio, which we term "rhabdom eccentricity," is independent of the size of the ommatidium. The data were analyzed with a Macintosh computer (Apple Computer, Cupertino, CA) using Engineering Tool Kit (Sof-Ware Tools, Boise, ID). For each time point, 20 ommatidia from 1 eye of 1 animal were measured.

Electrophysiology. In some experiments, one or both lateral optic nerves were exposed by removing a section of carapace and cutting. The distal end of a cut nerve was electrically stimulated to mimic efferent activity while the ERG (electroretinogram) of the innervated eye was monitored. Electrophysiological methods are described in detail elsewhere (Barlow, 1983). Briefly, the animal was clamped to a rigid platform in an aquarium located in a lightproof, shielded cage. The aquarium was filled with artificial seawater to the level of the lateral eye, and the gill structure was continuously washed with aerated seawater. The lateral eye ERG in response to a brief flash was recorded with a corneal electrode. Current pulses were delivered to the peripheral stump of the cut lateral optic nerve via a suction electrode and pulse stimulator (models PS1U6 and S44; Grass Instrument Co., Quincy, MA). The stimulation pattern of 2 pulses/ $/ \mathrm{sec}$ for $30 \mathrm{sec} / \mathrm{min}$ approximated that of endogenous efferent activity recorded at night (Barlow et al., 1977).

\section{Results}

\section{Diurnal rhythms in retinal structure}

Diurnal rhythms in the structure of the lateral eye retina were determined during a cloudless day and night at the end of June. Horseshoe crabs were taken directly from an ocean pen and fixed every $2 \mathrm{hr}$ over a period of $24 \mathrm{hr}$. Figure 2 shows the diurnal rhythms in aperture length, aperture diameter, and rhabdom eccentricity. In each case one $24 \mathrm{hr}$ cycle of data has been plotted twice. All 3 parameters change with time of day. The longer-lasting daytime changes reflect the long days and short nights at this time of the year. Table 1 (LD) gives summary data for these structural parameters.

Aperture length. The aperture begins to elongate at dawn and continues to do so until late afternoon, after which time it begins to shorten (Fig. 2.1). The shortening phase is more rapid than the lengthening phase, and commences in daylight in the late afternoon (1600-1800 hours). Aperture length is a dynamic parameter, which changes continuously during the daily cycle, except for a $6 \mathrm{hr}$ period at night (2200-0400 hours), when it is nearly at a minimum. In this experiment, the minimum mean aperture length was $1.1 \mu \mathrm{m}$, measured at 2400 hours; the max- 

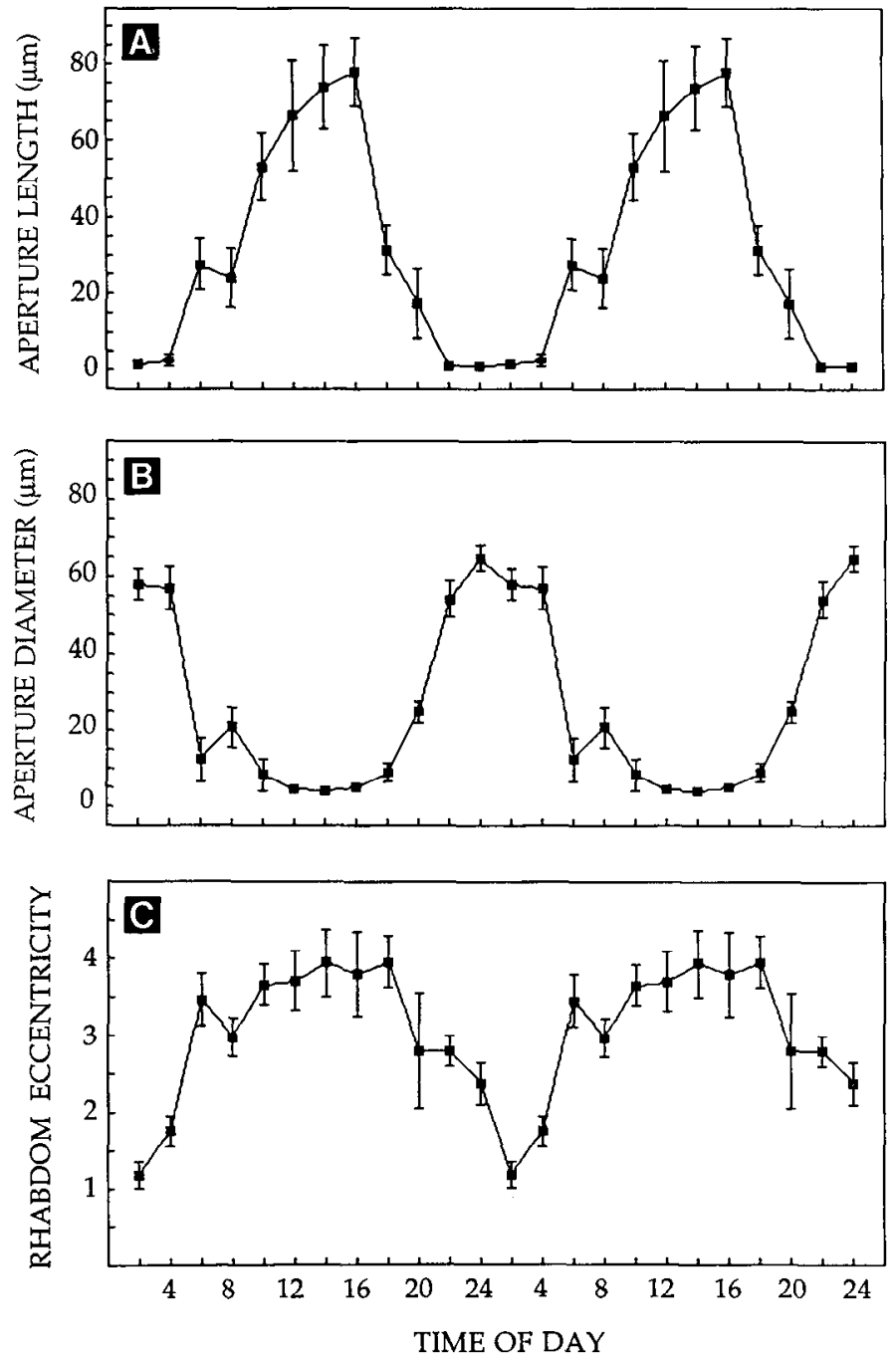

Figure 2. Daily variation of aperture length $(A)$, aperture diameter $(B)$, and rhabdom eccentricity $(C)$ in the lateral eyes of horseshoe crabs maintained in natural lighting. Animals were fixed on a clear day in late June. In this and the following figures, one $24 \mathrm{hr}$ set of data has been plotted twice to better show the pattern of variation with time. Time of day is reported as $24 \mathrm{hr}$ local clock time at Woods Hole, MA. Means \pm SD of 20 ommatidia.

imum mean aperture length was $78.1 \mu \mathrm{m}$, measured at 1600 hours. Thus in June, when daylight is longest, the diurnal variation in aperture length spans a range of $77 \mu \mathrm{m}$, and the longest apcrture has a length 71 timcs that of the shortest aperture.

Aperture diameter. The daily variation in the diameter of the aperture, measured at the midpoint of its length, has 2 intervals of relatively constant value separated by 2 transitional intervals (Fig. $2 B$ ). At dawn, the aperture rapidly narrows, achieving nearly its minimum diameter by 0600 hours, and it maintains this narrow value throughout the day (0600-1800 hours). In the evening (1800-2200 hours), the aperture widens to nearly its maximum diameter, which is maintained throughout the night (2200-0400 hours). In summary, the diameter of the aperture is dilated for $6 \mathrm{hr}$ at night, constricted for $12 \mathrm{hr}$ during the day, and in transition during the remaining $6 \mathrm{hr}$. In this experiment, the minimum mean aperture diameter was $4.1 \mu \mathrm{m}$, measured at 1400 hours; the maximum mean aperture diameter was 64.7 $\mu \mathrm{m}$, measured at 2400 hours. Thus in June, the diurnal variation
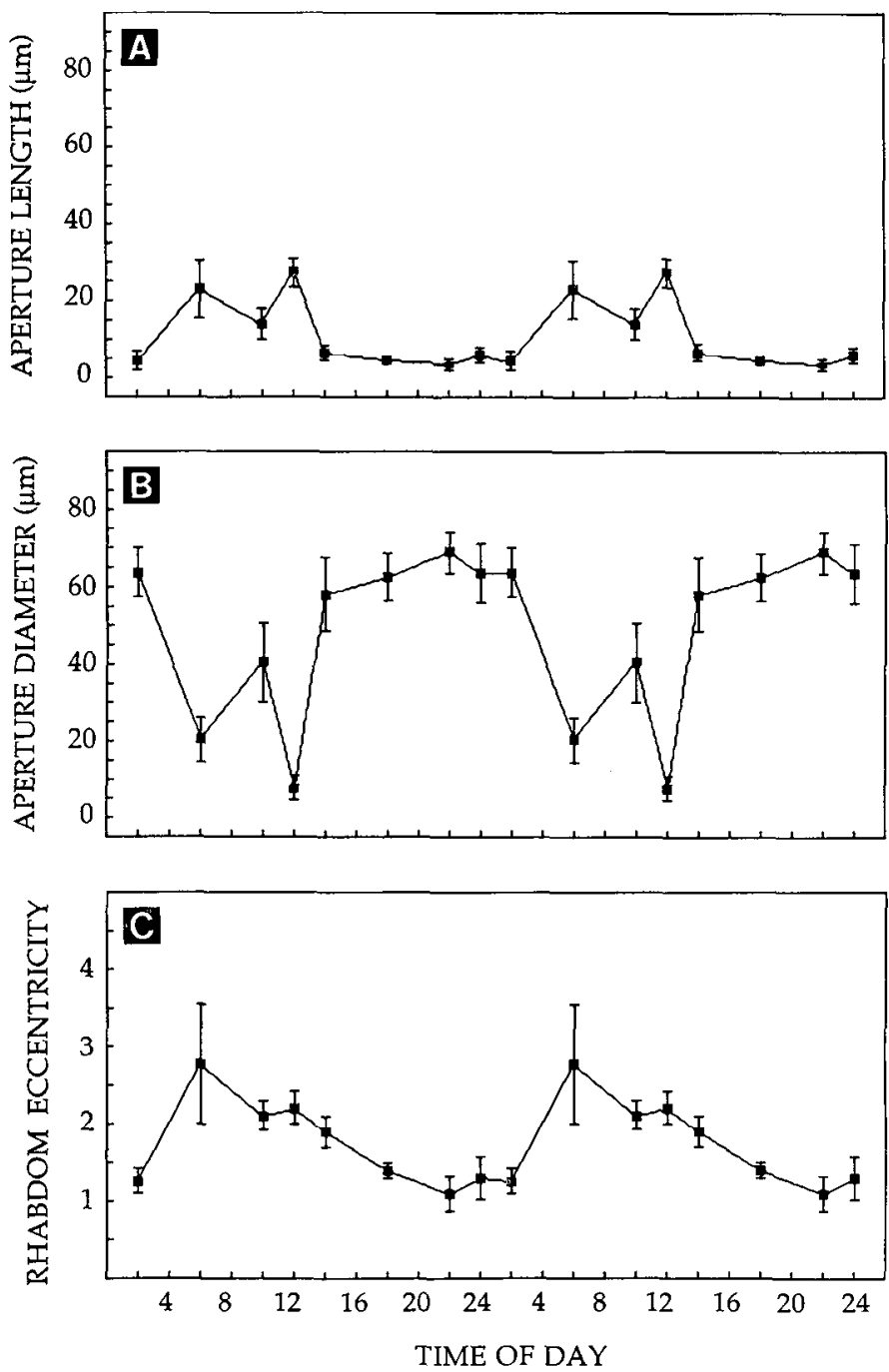

Figure 3. Circadian variation of aperture length $(A)$, aperture diameter $(B)$, and rhabdom eccentricity $(C)$ in the lateral eyes of horseshoe crabs maintained in constant darkness. Animals were fixed in late June. Means \pm SD of 20 ommatidia.

in aperture diameter spans a range of $60.6 \mu \mathrm{m}$, and the widest aperture has a diameter 15.8 times that of the narrowest.

Rhabdom eccentricity. At dawn the rhabdom changes from short and fat to long and thin (Fig. 2C). The eccentricity remains high during the day (0600-1800 hours) and then decreases again toward its minimum value, which is achieved in the early morning (0200 hours). In this experiment, the minimum mean rhabdom eccentricity was 1.17 , measured at 0200 hours; the maximum mean rhabdom eccentricity was 3.96 , measured at 1800 hours. Thus in June, the diurnal variation in rhabdom eccentricity spans a range of 2.79 , and the greatest eccentricity is 3.4 times that of the smallest.

\section{Circadian rhythms in retinal structure}

Circadian rhythms in retinal structure were determined on the same day in June with a second group of animals, placed in complete darkness in the early evening (1800 hours). Starting at midnight, the animals were fixed in total darkness at $4 \mathrm{hr}$ intervals, plus at midnight and noon, over a $24 \mathrm{hr}$ period. Figure 3 shows circadian rhythms in aperture length, aperture diameter, and rhabdom eccentricity. As before, one $24 \mathrm{hr}$ cycle of data 
Table 1. Summary of effects of circadian efferent activity and natural lighting on structural parameters in horseshoe crab retina

\begin{tabular}{|c|c|c|c|c|c|c|c|}
\hline \multirow[b]{2}{*}{ Parameter } & \multicolumn{2}{|c|}{ Max. value } & \multicolumn{2}{|c|}{ Min. value } & \multicolumn{2}{|c|}{ Range } & \multirow{2}{*}{$\begin{array}{l}\% \mathrm{LD} \text { range } \\
\text { of DD range }\end{array}$} \\
\hline & $\mathrm{DD}$ & LD & $\mathrm{DD}$ & LD & DD & LD & \\
\hline Aperture length & 3.4 & 1.1 & 27.3 & 78.1 & 23.9 & 77.0 & 322 \\
\hline Aperture diameter & 7.5 & 4.1 & 68.8 & 64.7 & 61.3 & 60.6 & 99 \\
\hline Rhabdom eccentricity & 1.1 & 1.2 & 2.7 & 4.0 & 1.6 & 2.8 & 175 \\
\hline
\end{tabular}

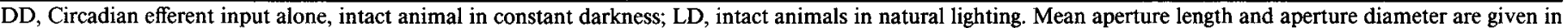
$\mu \mathrm{m}$. Mean rhabdom eccentricity is a dimensionless ratio: Rhabdom length divided by rhabdom width.

has been plotted twice. Table 1 (DD) gives summary data for these structural parameters.

Aperture length. In continuous darkness, the aperture lengthens between 0200 and 0600 hours, stays somewhat elongated through 1200 hours, shortens to nearly its minimum length by 1400 hours, and remains short until the following morning (Fig. $3 A$ ). The onset of lengthening in the morning occurs at the same time as that of the diurnal rhythm (Fig. $2 A$ ), but the increase is less than half that with natural lighting and lasts for a shorter duration. In this experiment, the minimum mean aperture length was $3.4 \mu \mathrm{m}$, measured at 2200 hours; the maximum mean aperture length was $27.3 \mu \mathrm{m}$, measured at 1200 hours. Thus the circadian variation in aperture length spans a range of $23.9 \mu \mathrm{m}$, and the longest aperture has a length 7 times that of the shortest aperture. In complete darkness, the amplitude of the daily rhythm in aperture length is only $31 \%$ of that occurring with natural lighting.

Aperture diameter. In continuous darkness, the changes in the diameter of the aperture mirror those in aperture length, but the size of the changes in aperture diameter is much greater than that in the changes in length. The aperture begins to narrow at about 0200 hours, reaches its minimum width at 1200 hours, returns to nearly its maximum width by 1400 hours, and remains at maximum diameter until the following morning (Fig. $3 B$ ). In this experiment, the minimum mean aperture diameter was $7.5 \mu \mathrm{m}$, measured at 1200 hours; the maximum mean aperture diameter was $68.8 \mu \mathrm{m}$, measured at 2200 hours. Thus the circadian variation in aperture diameter spans a range of $61.3 \mu \mathrm{m}$, and the widest aperture diameter is 9.6 times greater than the narrowest. In complete darkness, the range of values for aperture diameter is about the same as the range in diurnal lighting, although the endpoints are shifted about $4 \mu \mathrm{m}$ toward smaller values in natural lighting.

Rhabdom eccentricity. In continuous darkness, the shape of the rhabdom elongates and narrows between 0200 and 0600 hours and then slowly reverses these changes between 0600 and 2200 hours (Fig. 3C). Rhabdom eccentricity achieves a minimum value between 2200 and 0200 hours. In this experiment, the minimum mean rhabdom eccentricity was 1.10 , measured

Table 2. Comparison of effects of circadian efferent activity and optic nerve shock on structural parameters in horseshoe crab retina

\begin{tabular}{lll} 
Parameter & $\begin{array}{l}\text { Shocked/ } \\
\text { control }\end{array}$ & $\begin{array}{l}\text { Midnight/ } \\
\text { noon }\end{array}$ \\
\hline Aperture length $(\mu \mathrm{m})$ & 0.17 & 0.22 \\
Aperture diameter $(\mu \mathrm{m})$ & 8.54 & 8.42 \\
Rhabdom eccentricity & 0.68 & 0.61
\end{tabular}

The shocked and control eyes were both fixed at 11:45 in total darkness. The corresponding values for eyes maintained in continuous darkness were obtained at midnight and noon. at 2200 hours; the maximum mean rhabdom eccentricity was 2.74 , measured at 0600 hours. Thus the circadian variation in rhabdom eccentricity spans a range of 1.63 , and the greatest eccentricity is 2.5 times that of the smallest. In constant darkness, the amplitude of the rhythm in rhabdom eccentricity is only $57 \%$ of the amplitude in cyclic lighting.

\section{Control mechanisms: circadian efferent input}

Blocking the efferent input by cutting the optic nerve abolishes the endogenous circadian rhythm in the ERG. Shocking the distal stump of the cut lateral optic nerve to mimic normal efferent activity increases the ERG response to near normal nighttime values (Barlow et al., 1977). Quantitative modeling of photon catch indicates that changes in the ERG arise from changes in aperture dimensions and rhabdom eccentricity (Chamberlain and Fiacco, 1985). Acutely blocking the efferent input by nerve section thus eliminates the circadian rhythm in ERG by eliminating the circadian rhythm in retinal structure. Sectioning the optic nerve at noon leaves the structure of the retina in the daytime state, regardless of when, in the next 24 $\mathrm{hr}$, the eye is fixed. The aperture remains long and narrow and the rhabdom eccentricity remains high. Shocking the distal stump of the cut optic nerve moves the structure toward the nighttime state.

For example, in one experiment, a lateral optic nerve was cut at 1000 hours and, beginning at 1100 hours, it was shocked for 45 min while the ERG responses of both eyes were monitored. The ERG response of the intact eye remained unchanged, while that of the shocked eye increased to 3.5 times its starting value. Both eyes were fixed at 1145 hours. Table 2 gives the resulting changes in structural parameters for this experiment and the comparable circadian changes. When the increase in ERG due to shocking corresponds to that caused by the onset of natural efferent activity (Barlow, 1983), the changes in the length and diameter of the aperture are comparable, but the change in rhabdom eccentricity caused by shocking the cut optic nerve is less than that produced by nighttime efferent activity. Similar results were obtained when the optic nerve was cut during the day and shocked at night. In sum, shocking the cut optic nerve moves the ommatidial structure toward the nighttime state whether the experiment is performed during the day or at night.

\section{Control mechanisms: circadian efferent input plus natural lighting}

Natural lighting versus continuous darkness. Natural lighting enhances the circadian rhythm in the structure of the retina. The effects of natural lighting are shown in Figure 4 by the differences between the diurnal curves of Figure 2 and the circadian curves of Figure 3. In general, daylight increases aperture length, decreases aperture width, and increases rhabdom eccentricity. There are times of day when the values measured in continuous dark- 

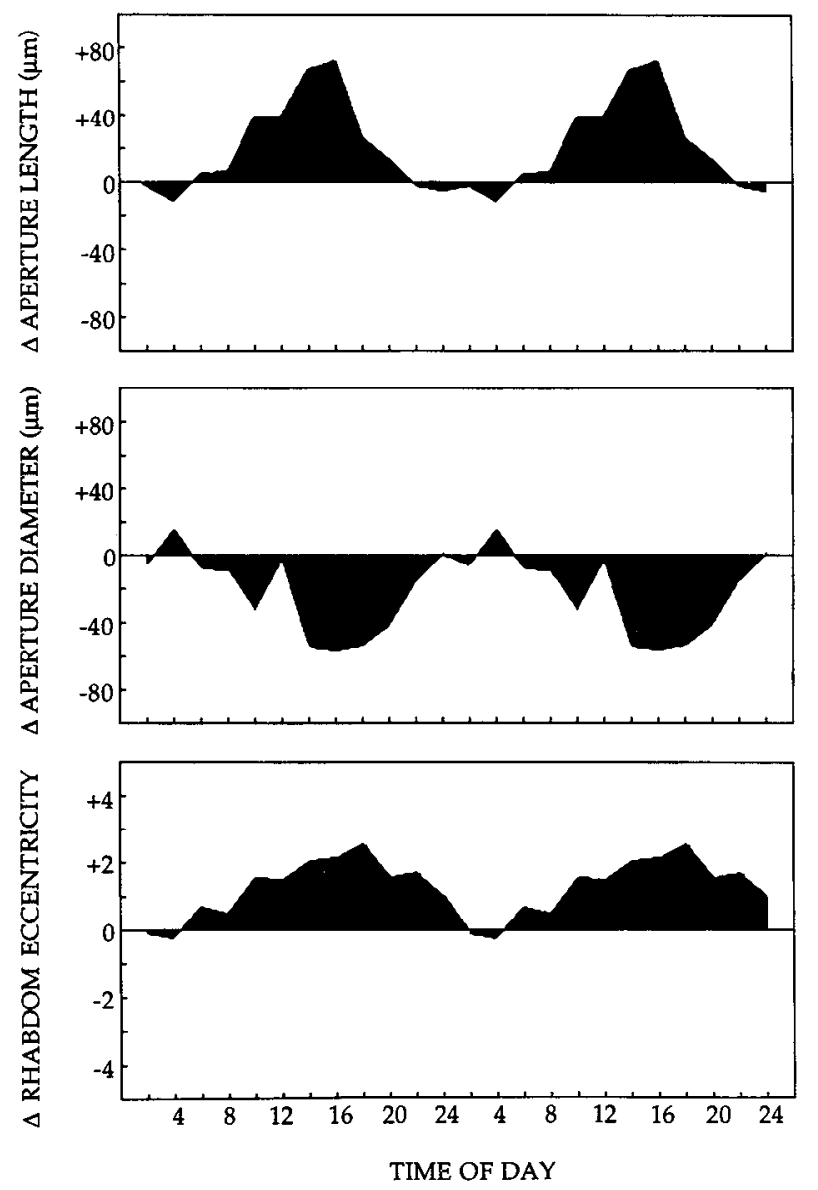

Figure 4. Enhancement of circadian rhythms in lateral eye structure by natural lighting. The difference between measured parameters in natural lighting (Fig. 2) and in constant darkness (Fig. 3) is shown.

ness are nearly minimum, and those measured under diurnal lighting are nearly maximum. Thus, daylight causes changes in each parameter over almost its full range of values. Furthermore, the effects of daylight appear to continue into the night. The lingering effects of daylight are most extensive for rhabdom eccentricity and least extensive for aperture length.

Although the largest effects of daylight are the lengthening and narrowing of the aperture and rhabdom, smaller reverse effects occur at night. These effects are represented by the regions of negative values of $\Delta$ aperture length and $\Delta$ rhabdom eccentricity, and of positive values of $\Delta$ aperture diameter in Figure 4.

A comparison of Table 1 with Figure 4 reveals that (1) between 2200 and 0400 hours, the aperture is shorter in natural lighting than in constant darkness (significant at the $0.1 \%$ level), and the absolute minimum value of aperture length is achieved in diurnal lighting; (2) the absolute maximum value of aperture diameter occurs in constant darkness, but at around 0400 hours the aperture is wider in natural lighting than in constant darkness (significant at the $1 \%$ level); and (3) the absolute minimum value of rhabdom eccentricity occurs in constant darkness, but at around 0400 hours the rhabdom is shorter and fatter in natural lighting than in constant darkness (significant at the 5\% level). In comparison to circadian changes in constant darkness, a greater shortening at night accompanies the light-driven lengthening of the aperture during the day. Likewise, the greater narrowing of
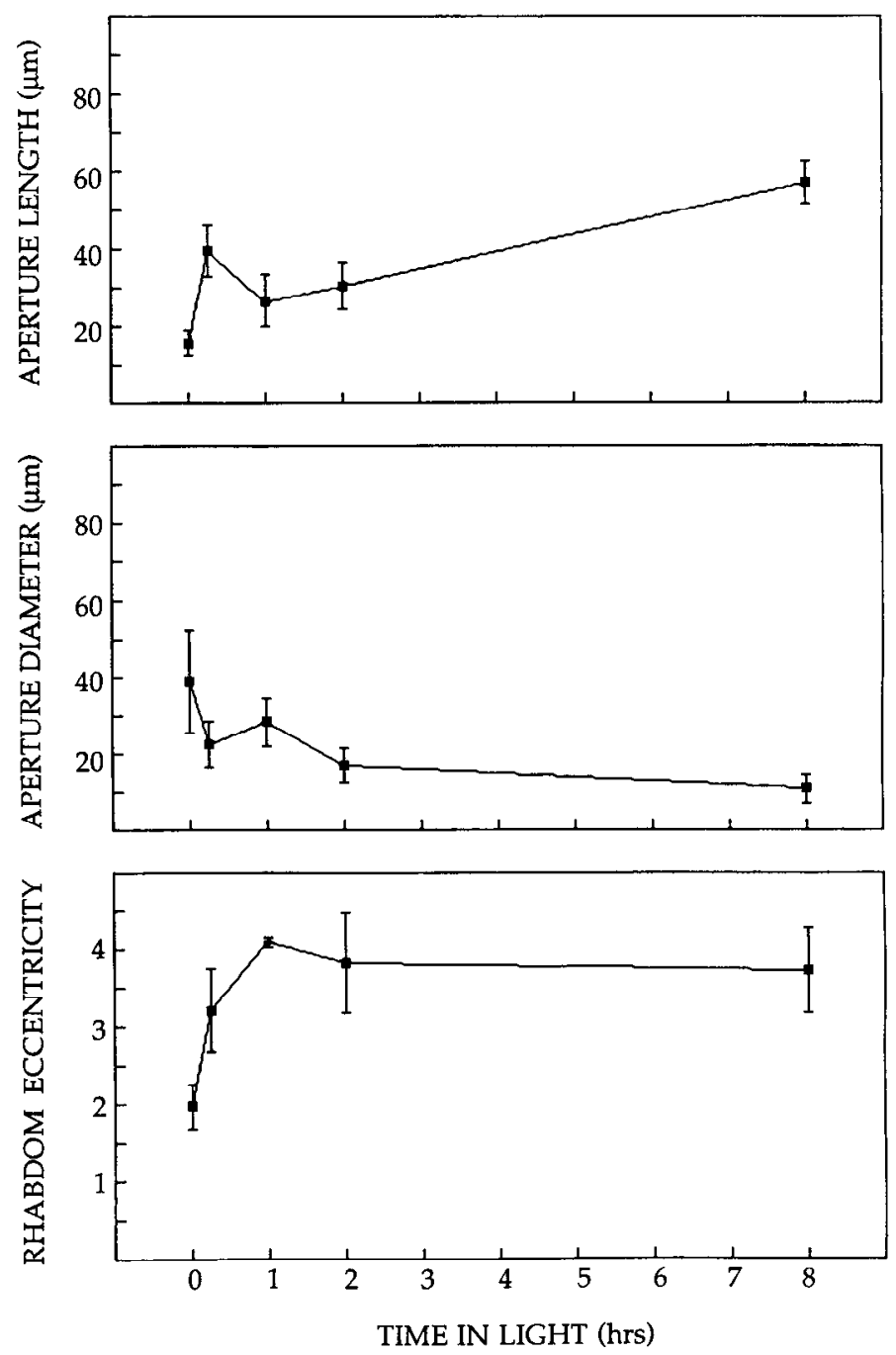

Figure 5. Effect of length of light exposure on the structure of the lateral eye. Animals were taken from darkness, exposed to daylight for various durations, and fixed in daylight at 1600 hours, local time.

the aperture during daylight is accompanied by greater widening at night. Natural lighting causes the rhabdom to be longer and thinner during the day and shorter and fatter at night than is the case in continuous darkness.

Effects of lengthening light exposure. The effects of progressive light exposure on the structure of the retina were determined at 1600 hours, when light-driven changes have their greatest magnitude (Fig. 4). For this experiment, animals were placed in darkness in the evening, brought into daylight at staggered times, and were all fixed in daylight at 1600 hours on the following day. Figure 5 shows the average measured changes in aperture length, aperture diameter, and rhabdom eccentricity for light exposures up to $8 \mathrm{hr}$. Although the changes in all 3 parameters are graded by the duration of light exposure, the time courses are different. Rhabdom eccentricity reaches its maximum lightdriven value after a $1 \mathrm{hr}$ exposure to daylight. The aperture continues to lengthen even after $8 \mathrm{hr}$ of light exposure without apparent saturation. The diameter of the aperture progressively declines over the $8 \mathrm{hr}$ interval and appears to be reaching saturation.

These results relate to the general shape of the light-driven increases in aperture length (Fig. $2 A$ ) and rhabdom eccentricity 
(Fig. 2C). The slow, graded, nonsaturating response of aperture length to light exposure is consistent with the gradually rising leg of the asymmetrical daytime increase in aperture length during the diurnal cycle, which suggests that the shape of the diurnal rhythm in aperture length largely reflects the dynamics of the structural responses to light. By contrast, the rapidly saturating light response of rhabdom eccentricity results in a symmetrical daytime increase in that parameter during the diurnal cycle, probably reflecting the pattern of light intensity.

\section{Control mechanisms: natural lighting in the absence of efferent input}

The effects of natural lighting alone were investigated by blocking the efferent input with optic nerve section. The lateral optic nerves of a group of animals were bilaterally cut at noon l week before fixation. The animals were maintained in the same conditions as intact animals. Horseshoc crabs wcrc taken directly from an ocean pen and fixed every $4 \mathrm{hr}$, plus at noon and midnight, for one $24 \mathrm{hr}$ period of clear weather at the end of June. Figure 6 shows the values of aperture length, aperture diameter, and rhabdom eccentricity under these conditions, plotted with the normal diurnal rhythms from Figure 2. Although the values of all 3 parameters are not constant over 24 hr they do not show a pattern indicative of photomechanical changes induced by natural lighting.

Aperture length. The minimum mean aperture length was 29.6 $\mu \mathrm{m}$, measured at 1200 hours; the maximum mean aperture length was $64.2 \mu \mathrm{m}$, measured at 1400 hours (Fig. $6 \mathrm{~A}$ ). In the absence of circadian efferent inputs to the retina, aperture length under natural lighting shows variations that span a range of 34.6 $\mu \mathrm{m}$, and the length of the longest aperture is about 2 times the length of the shortest aperture. Removing efferent activity affects apcrturc length in 3 significant ways under conditions of natural lighting. First, there is no obvious diurnal rhythm in aperture length - the maximum and minimum values measured were only $2 \mathrm{hr}$ apart. Second, the range of values is reduced and is offset toward longer apertures. Third, short apertures, typical of nighttime both under natural lighting and constant darkness, were never observed. To a first approximation, the aperture length is constant during the diurnal cycle, with an average value of $43.5 \mu \mathrm{m}$.

Aperture diameter. The minimum mean aperture diameter was $4.6 \mu \mathrm{m}$, measured at 1400 hours; the maximum mean aperture diameter was $19.7 \mu \mathrm{m}$, measured at 1200 hours (Fig. $6 B$ ). In the absence of circadian efferent inputs to the retina, aperture diameter under natural lighting shows variations that span 15.1 $\mu \mathrm{m}$ and the diameter of the widest aperture is about 4 times the diameter of the narrowest aperture. Removing efferent activity has 3 major effects on aperture width under conditions of natural lighting. First, there is no obvious diurnal rhythm in aperture diameter - the maximum and minimum values were separated by only $2 \mathrm{hr}$. Second, the range of values is greatly reduced and is offset toward narrower apertures. Third, wide apertures, typical of nighttime both under natural lighting and constant darkness, were never observed. To a first approximation, the aperture diameter is constant during the diurnal cycle, with an average value of $9.5 \mu \mathrm{m}$.

Rhabdom eccentricity. The minimum mean rhabdom eccentricity was 1.66 , measured at 2400 hours; the maximum mean rhabdom eccentricity was 2.63 , measured at 2200 hours (Fig. $6 C$ ). In the absence of circadian efferent inputs to the retina, rhabdom eccentricity under natural lighting shows variations
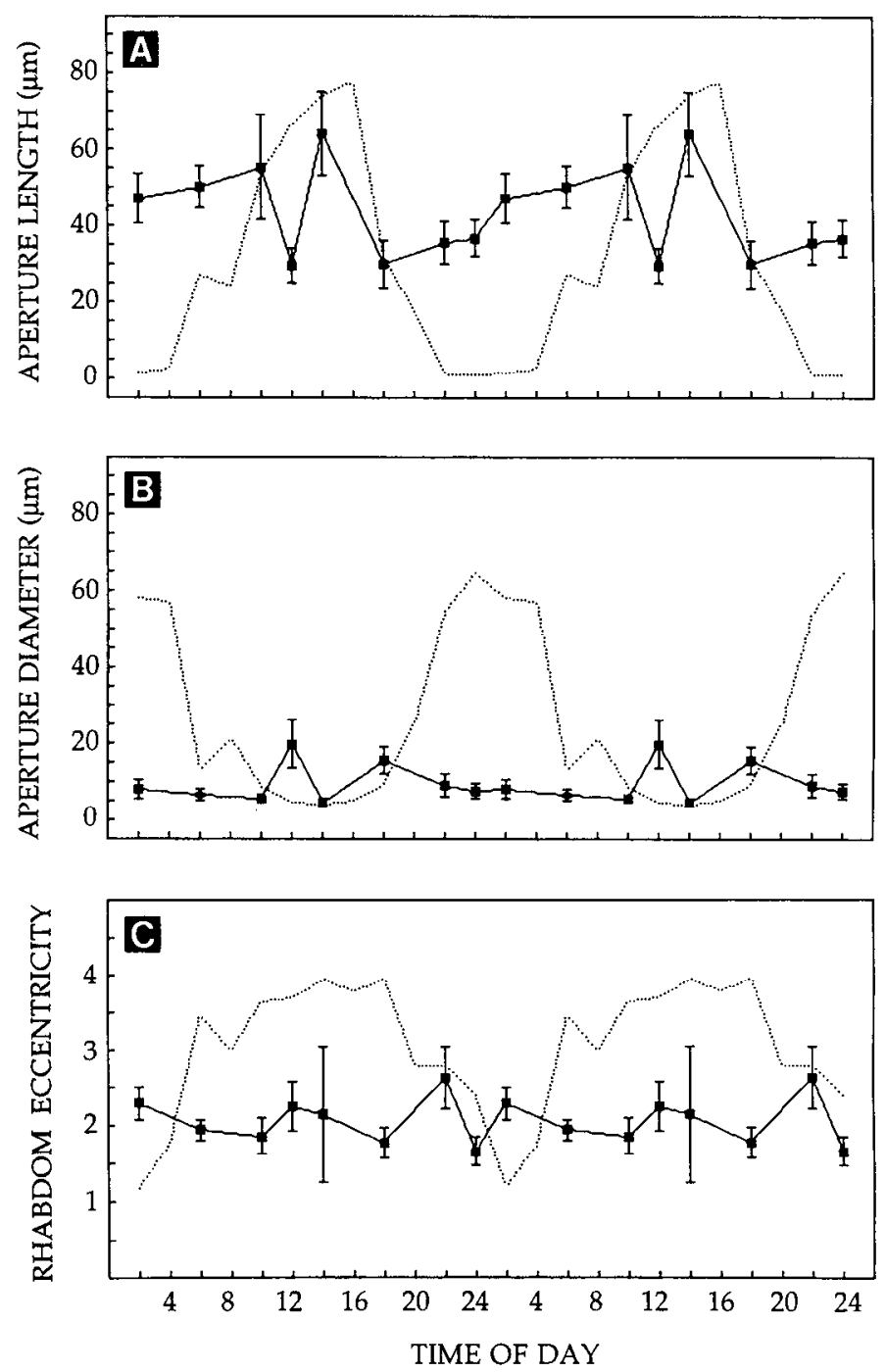

Figure 6. Effect of natural lighting on aperture length $(A)$, aperture diameter $(B)$, and rhabdom eccentricity $(C)$ of the lateral eyes of animals with bilaterally cut lateral optic nerves (solid curves) and of intact animals (dotted lines). In the absence of circadian efferent input, no cyclic variation in these structural parameters appears to be correlated with natural lighting. Animals were fixed on a clear day in late June. Means \pm SD of 20 ommatidia.

spanning a range of 0.97 , and the greatest eccentricity is about 1.6 times the smallest. Removing efferent activity has 3 notable effects on rhabdom eccentricity under conditions of natural lighting. First, there is no obvious diurnal rhythm in rhabdom eccentricity - the maximum and minimum values were only 2 $\mathrm{hr}$ apart. Second, the range of valucs is reduccd and is offset toward lower values. Third, large eccentricities, typical of daytime retinas under natural lighting, were never observed. To a first approximation, the rhabdom eccentricity is constant during the diurnal cycle, with an average value of 2.07 .

In summary, without circadian efferent inputs, retinal ommatidia of animals maintained in natural lighting have long, narrow apertures and relatively short and fat rhabdoms. Note that this combination of parameters is never observed in intact animals, whether they are exposed to natural lighting or maintained in constant darkness. Natural lighting, in the absence of circadian efferent input from the brain to the retina, does not induce a clear diurnal rhythm in aperture length, aperture diameter, or rhabdom eccentricity. 


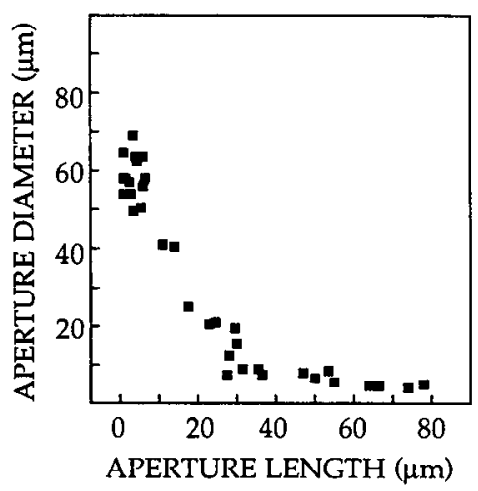

Figure 7. Relationship between mean aperture diameter and mean aperture length for all the animals in this study. Each point is the mean of measurements of 20 ommatidia.

\section{Further analysis of structural parameters}

Aperture length and diameter. Our analysis of serial sections suggests that the aperture changes shape as it changes its dimensions. For example, for intact animals in natural lighting, the aperture progressively lengthens in the interval from 1200 to 1600 hours (Fig. $2 A$ ), even though the aperture diameter is essentially constant during this same time period (Fig. $2 B$ ). Figure 7 shows the relationship between mean aperture diameter and mean aperture length for all experiments. There is a strong correlation between the 2 parameters: wide apertures are short, and narrow apertures are long, but the relationship is complex. Short apertures are shaped like a cylinder and changes in length and diameter appear to be reciprocal, preserving the volume of the cylinder. Longer apertures are not shaped like a cylinder. Their diameters are consistently smaller than would be expected, and fall further and further behind the calculated value as aperture length increases. This departure is due to the necessary boundary conditions at the ends of the aperture. Both the distal tip of the cuticular cone and the proximal tip of the rhabdom constrain the minimum diameter of the aperture at the ends. These changes are summarized in Figure 8, which shows idealized longitudinal, midaperture sections. In Figure $8 A$, the aperture is $5 \mu \mathrm{m}$ long and $60 \mu \mathrm{m}$ in diameter. In Figure $8 B$, it is still essentially cylindrical, $11 \mu \mathrm{m}$ long and $40 \mu \mathrm{m}$ in diameter. In Figure $8, C, D$, the ends have reached their minimum diameter, which is greater than that calculated for a cylinder of constant volume (dotted lines), while the diameter in the middle of the length is less than that calculated. Figure $8 C$ shows an aperture $30 \mu \mathrm{m}$ long and $15 \mu \mathrm{m}$ in diameter at the midpoint. Figure $8 D$ shows an aperture $70 \mu \mathrm{m}$ long and $5 \mu \mathrm{m}$ in diameter at the midpoint. The change in shape of long apertures is probably sufficient to explain the departure of the length-diameter

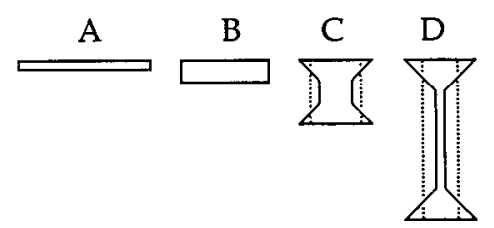

Figure 8. Stylized scale diagram of the shape changes that accompany changes in the measured dimensions of the aperture. Dotted lines indicate the predicted diameters if the aperture were a uniform cylinder of constant volume. $A, 5 \mu \mathrm{m}$ long, $60 \mu \mathrm{m}$ wide. $B, 11 \mu \mathrm{m}$ long, $40 \mu \mathrm{m}$ wide. $C, 30 \mu \mathrm{m}$ long, $15 \mu \mathrm{m}$ wide. $D, 70 \mu \mathrm{m}$ long, $5 \mu \mathrm{m}$ wide.

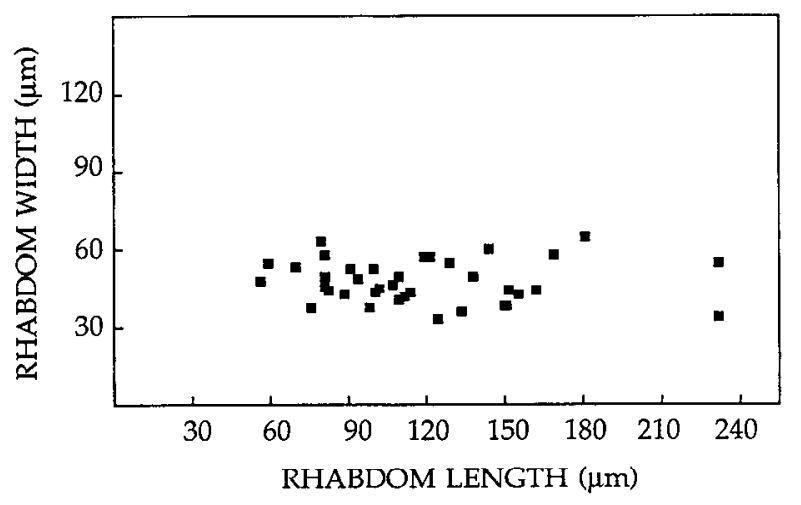

Figure 9. Relationship between mean rhabdom width and mean rhabdom length for all the animals in this study. The range of values is due to variations in ommatidial size. Rhabdom eccentricity, the ratio of length to width, is a more sensitive measure of rhabdom shape. Each point is the mean of measurements of 20 ommatidia.

relationship from that of a cylinder of constant volume, without having to invoke actual changes in aperture volume.

Rhabdom length and width. Figure 9 shows the relationship between mean rhabdom width and mean rhabdom length for all experiments. The parameter we have reported, rhabdom eccentricity, is the ratio of length to width. For any rhabdom length, a range of rhabdom widths, and thus eccentricities, is possible. Width values appear to be independent of length values. Data from a single retina show that longer rhabdoms are wider. We conclude that ommatidial size, which can be quite variable within a retina, is a major source of variation in rhabdom width or length measurements, and that the dimensionless quantity, rhabdom eccentricity, is a more appropriate measure of ommatidial structure.

\section{Summary}

Structural rhythms are pronounced in the Limulus lateral eye in situ under natural cyclic lighting. These diurnal rhythms result from underlying circadian rhythms driven by efferent activity from a circadian clock in the brain and enhanced by natural lighting. The extremes of ommatidial morphology in constant darkness and natural lighting are summarized in Figure 10. The dual effects of circadian efferent activity and cyclic lighting are not simply additive, because blocking the circadian input by optic nerve section eliminates the effects of cyclic lighting. These interactions are summarized in Figure 11.

\section{Discussion}

The circadian efferent input to the Limulus lateral eye has a triple role in adapting the structure of the retina for the cyclic lighting of the animal's environment. First, the onset of efferent input at dusk triggers nighttime structural changes and its offset before dawn allows the structure to return to the daytime state in darkness. Second, circadian nighttime efferent input primes the photomechanical movements triggered by daylight. Third, it enables the effects of daylight to enhance the nighttime changes. The nighttime changes appear to optimize retinal function for low levels of illumination, and the daytime changes appear to adapt the retina for bright daytime illumination.

\section{Circadian efferent input versus natural lighting}

Circadian efferent inputs to the retina produce structural rhythms in the absence of natural lighting. Natural lighting synchronizes 


\section{Nighttime \\ Darkness}

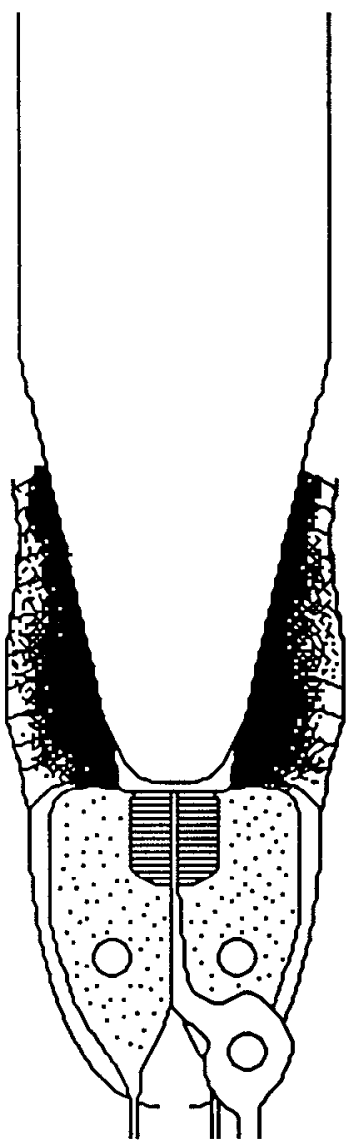

Daytime

Darkness

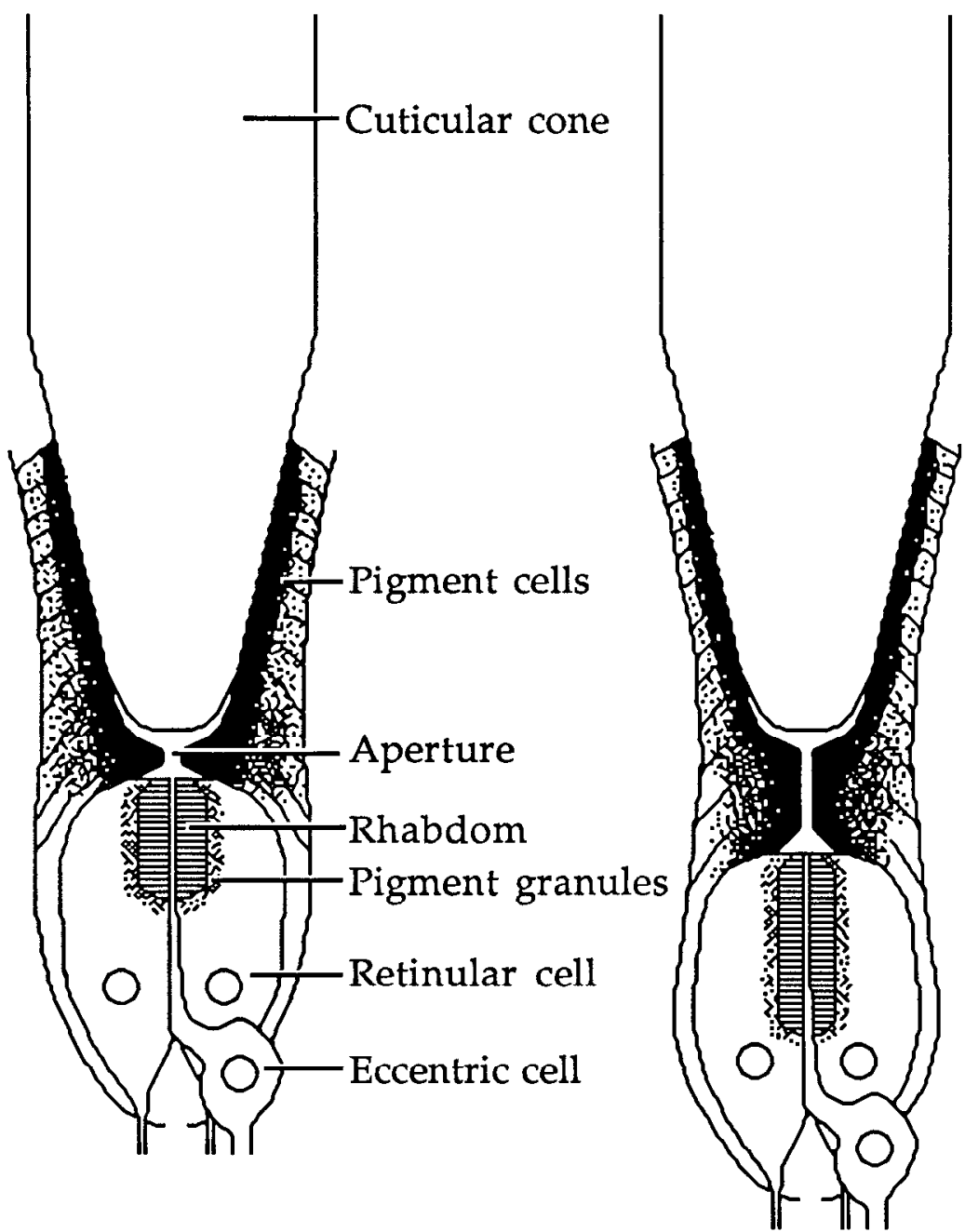

Figure 10. Summary longitudinal drawings of the structure of Limulus ommatidia at the extremes of circadian (nighttime darkness and daytime darkness) and diurnal (nighttime darkness and daytime daylight) rhythms in retinal structure. The drawings are approximately to scale. The differences between the nighttime structure in natural lighting and that in constant darkness cannot be represented at this magnification.

the circadian rhythms, preparing the retina for photomechanical movements driven by daylight. These photomechanical movements require efferent input the previous night. Apparently, the nighttime efferent input primes the retina for photomechanical changes that occur during the day, when the efferents are silent (Barlow, 1983). This effect appears to be analogous to the efferent priming of daily membrane shedding from the rhabdom (Chamberlain and Barlow, 1979, 1984). The structural changes of the retina reported here for natural lighting and constant darkness confirm and further elucidate our earlier conclusions based on ERG recordings and the morphology of dark-adapted eyes (Barlow et al., 1977; Chamberlain and Barlow, 1977).

Cyclic lighting has long been thought to serve as a Zeitgeber that synchronizes the phase of a circadian rhythm (for a review, see Pittendrigh, 1981). Our results indicate that cyclic lighting also has an integral role in the expression of the rhythm itself. Cyclic lighting magnifies the structural changes observed both day and night in constant darkness. The endogenous daytime changes are enhanced by photomechanical movements and the endogenous nighttime changes are enhanced by the preceding

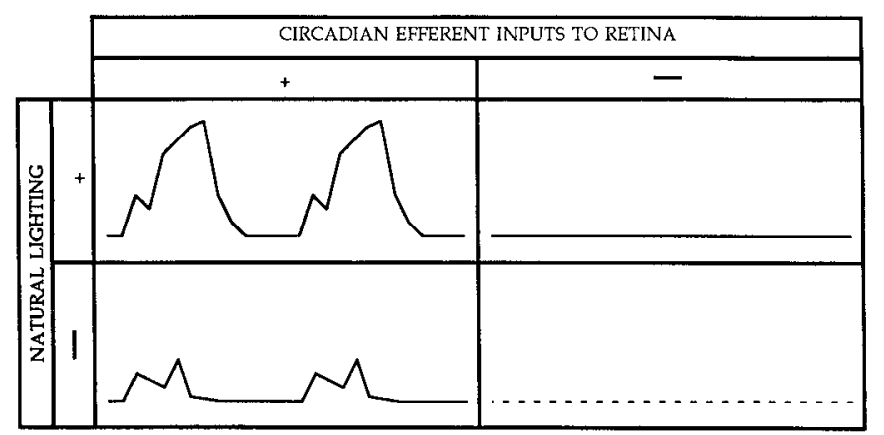

Figure 11. Summary of the interactions of circadian efferent activity and natural lighting in controlling retinal structure. Cyclic lighting and efferent optic nerve activity together produce maximal structural rhythms in the Limulus lateral eye. Circadian efferent activity alone produces a reduced structural rhythm. Natural lighting alone produces no structural rhythm. Dotted line represents the lack of structural rhythm in the absence of both natural lighting and efferent activity, inferred from the constant electroretinographic response measured under such conditions. Aperture length is the structural parameter used for this summary. 
period of daylight. The enhanced changes at night are small but significant. They are reminiscent of the effects of cyclic lighting on the synthesis of $N$-acetyltransferase by isolated pineal organs (Takahashi et al., 1980) and by isolated pineal cells (Robertson and Takahashi, 1985). The enhancing effects of cyclic lighting both in the chick pineal and the Limulus eye may result from the direct effects of light during the day and from the synchronization of circadian oscillators with different endogenous periods at night.

\section{Cellular mechanisms for ommatidial movement}

Both light and efferent activity can activate ommatidial movements in the Limulus lateral eye. The retina's response to the efferent input appears to be mediated by efferent neurotransmitter(s), one of which is octopamine. We do not know as yet how the response to light is mediated. It does not appear to involve immediate efferent feedback because the efferents are silent during the day (Barlow, 1983). It is likely that both light and efferent input use a common final pathway, although their combined effects are not additive. Efferent activity at night somehow primes photomechanical movements during the day, and daylight somehow enhances the effect of nighttime efferent input.

Several lines of evidence suggest that the mechanisms that control the shape of the aperture and those that alter rhabdom eccentricity may be different. The diurnal rhythms in the aperture length and rhabdom eccentricity of intact animals have different forms (compare Fig. 3, $A$ and $C$ ). During progressive light exposure, the time courses of photomechanical changes of aperture length and rhabdom eccentricity are quite different (Fig. 5). Also, the combination of long apertures and intermediate rhabdom eccentricities measured for animals with chronically cut optic nerves is never achieved in intact animals in natural lighting or constant darkness.

Microtubules and microfilaments appear to mediate the changes in retinal structure. These cytoskeletal elements were implicated in a preliminary study using the microtubule inhibitors vinblastine, vincristine, podophyllotoxin, and colchicine and the microfilament inhibitor cytochalasin B (Barlow and Chamberlain, 1980b). Of special interest was our finding that, while all of these agents decreased the ERG response at night and prevented the full development of the aperture changes characteristic of nighttime, they also increased the ERG response during the day, with the corresponding structural changes. Whether an inhibitor is administered during the day or at night, the same final structural state is achieved. This suggests that an active, push-pull cytoskeletal mechanism is responsible for producing both the daytime and nighttime structures.

\section{Temporal effects of efferent input}

The multiple effects of the circadian efferent input to the retina have different dynamics, and the essential dichotomy between immediate and delayed effects is now quite clear. Upon the initiation or cessation of efferent activity, changes in photon catch, acceptance angle, photoreceptor noise, photoreceptor gain, and lateral inhibition begin immediately. Changes in photoreceptor noise and gain are detectable within seconds of the onset of efferent activity (Barlow et al., 1985, 1987). Changes in photon catch and acceptance angle are caused by the changes in ommatidial structure reported here and proceed more slowly. The effects of these structural changes can be detected in ERG recordings about 4 min after the onset of efferent activity, and are complete in about 50 min (Barlow et al., 1980; Barlow, 1983).

By contrast, the effects of efferent activity on microvillar membrane shedding (Chamberlain and Barlow, 1979, 1984) and photomechanical movements are delayed. In the natural environment, both of these adaptive processes are primed by efferent activity at night and initiated hours later by daylight. In summary, the clock's multiple effects on the retina begin within seconds of efferent onset and extend over the entire day, even after efferent activity has ceased.

\section{Comparison with control of retinomotor movements in the teleost retina}

Prominent structural rhythms in the retinas of various species of fish have been described and investigated (see Burnside and Nagle, 1983, for a review). Although the details of the processes differ, a comparison of the overall strategies that have evolved in fish and horseshoc crabs revcals some interesting similarities. In both animals, the structural rhythms serve to adapt the retina for cyclic lighting. In both, there is an underlying circadian rhythm of structural changes in continuous darkness that is accentuated by natural light (Levinson and Burnside, 1981). In both, the structural adaptations for daylight begin before actual dawn (Burnside and Ackland, 1984). In fish, however, the circadian oscillator(s) appear to be within the eye (Dearry and Barlow, 1987), whereas in Limulus it is in the brain (Barlow et al., 1977).

Across species, the designs of systems that control circadian rhythms appear to be quite diverse. Evidence for intraocular circadian oscillators has been reported in rat (LaVail and Ward, 1978; Goldman et al., 1980; Wirz-Justin et al., 1984; Terman and Terman, 1985), Xenopus (Besharse and Iuvone, 1983; Flannery and Fisher, 1984), Aplysia (Jacklet, 1969), and Bulla (Block and Wallace, 1982). Evidence for central control of retinal circadian rhythms has been reported in rat (Teirstein et al., 1980), rabbit (Brandenberg et al., 1981), lizard (Fowlkes et al., 1985), fish (Dearry and Barlow, 1987), Limulus (Barlow et al., 1977), and scorpion (Fleissner and Fleissner, 1978). In some species, such as rat, lizard, and fish, a retinal circadian oscillator is modulated by input from the central nervous system. In others, such as rabbil, Limulus, and scorpion, the circadian oscillator is located in the central nervous system and its influence is transmitted to the retina via efferent optic nerve fibers. Whatever the distribution of components in the nervous system, circadian rhythms appear to be important adaptive processes in vision.

\section{References}

Barlow, R. B., Jr. (1983) Circadian rhythms in the Limulus visual systcm. J. Ncurosci. 3: 856-870.

Barlow, R. B., Jr., and S. C. Chamberlain (1980a) Light and a circadian clock modulate structure and function in Limulus photoreceptors. In The Effects of Constant Light on Visual Processes, T. P. Williams and B. N. Baker, eds., pp. 247-269, Plenum, New York.

Barlow, R. B., Jr., and S. C. Chamberlain (1980b) Microtubule inhibitors can increase the sensitivity of the Limulus eye. Invest. Ophthalmol. Vis. Sci. (Suppl.) 19: 245.

Barlow, R. B., Jr., S. J. Bolanowski, Jr., and M. L. Brachman (1977) Efferent optic nerve fibers mediate circadian rhythms in the Limulus eye. Science 197: 86-89.

Barlow, R. B., Jr., S. C. Chamberlain, and J. Z. Levinson (1980) Limulus brain modulates structure and function of the lateral eyes. Science 210: $1037-1039$. 
Barlow, R. B., Jr., E. Kaplan, G. H. Renninger, and T. Saito (1985) Efferent control of circadian rhythms in the Limulus lateral eye. Neurosci. Res. (Suppl.) 2: S65-S78.

Barlow, R. B., Jr., E. Kaplan, G. H. Renninger, and T. Saito (1987) Circadian rhythms in Limulus photoreceptors. I. Intracellular recordings. J. Gen. Physiol. 89: 353-378.

Batra, R. (1983) Efferent control of visual processing in the lateral eye of the horseshoe crab. In Special Report ISR-S-22, Institute for Sensory Research, Syracuse University, Syracuse, NY.

Batra, R., and R. B. Barlow, Jr. (1982) Efferent control of pattern vision in Limulus. Soc. Neurosci. Abstr. 8: 49.

Battelle, B.-A., and J. A. Evans (1984) Octopamine release from centrifugal fibers of the Limulus peripheral visual system. J. Neurochem. 42: 71-79.

Battelle, B.-A., J. A. Evans, and S. C. Chamberlain (1982) Efferent fibers to Limulus eyes synthesize and release octopamine. Science 216: 1250-1252.

Behrens, M. E. (1974) Photomechanical changes in the ommatidia of the Limulus lateral eye during light and dark adaptation. J. Comp. Physiol. 89: 45-57.

Besharse, J., and M. Iuvone (1983) Circadian clock in Xenopus eye controlling retinal serotonin $N$-acetyltransferase. Nature 305 : $133-$ 135.

Block, G., and S. Wallace (1982) Localization of a circadian pacemaker in the eye of a mollusc, Bulla. Science 217: 155-157.

Brandenburg, J., A. Bobbert, and F. Eggelmeyer (1981) Evidence for the existence of a retino-hypothalamo-retinal loop in rabbits. Int. J. Chronobiol. 8: 13-29.

Burnside, B., and N. Ackland (1984) Effects of circadian rhythm and cAMP on retinomotor movements in the green sunfish, Lepomis cyanellus. Invest. Ophthalmol. Vis. Sci. 25: 539-545.

Burnside, B., and B. W. Nagle (1983) Retinomotor movements of photoreceptors and retinal pigment epithelium: Mechanisms and regulation. In Progress in Retinal Research, N. Osborne and G. Chader, eds., pp. 67-109, Pergamon, New York.

Chamberlain, S. C., and R. B. Barlow, Jr. (1977) Morphological correlates of efferent circadian activity and light adaptation in the Limulus lateral eye. Biol. Bull. 153: 418-419.

Chamberlain, S. C., and R. B. Barlow, Jr. (1979) Light and efferent activity control rhabdom turnover in Limulus photoreceptors. Science 206: 361-363.

Chamberlain, S. C., and R. B. Barlow, Jr. (1981) Modulation of retinal structure in Limulus lateral eye: Interactions of light and efferent inputs. Invest. Ophthalmol. Vis. Sci. (Suppl.) 20: 75.

Chamberlain, S. C., and R. B. Barlow, Jr. (1984) Transient membrane shedding in Limulus photoreceptors: Control mechanisms under natural lighting. J. Neurosci. 4: 2792-2810.

Chamberlain, S. C., and P. A. Fiacco (1985) Models of circadian changes in Limulus ommatidia: Calculations of changes in acceptance angle, quantum catch, and quantum gain. Invest. Ophthalmol. Vis. Sci. (Suppl.) 26: 340.

Dearry, A., and R. B. Barlow, Jr. (1987) Circadian rhythms in the green sunfish retina. J. Gen. Physiol. (in press).

Engbretson, G. A., and S. C. Chamberlain (1981) Efferent fibers to the Limulus lateral eye may contain the neuropeptide ranatensin. Invest. Ophthalmol. Vis. Sci. (Suppl.) 20: 75.
Fahrenbach, W. H. (1973) The morphology of the Limulus visual system $\mathrm{V}$. Protocerebral neurosecretion and ocular innervation. $\mathrm{Z}$. Zellforsch. 144: 153-166.

Fahrenbach, W. H. (1981) The morphology of the Limulus visual system VII. Innervation of photoreceptor neurons by neurosecretory efferents. Cell Tissue Res. 216: 655-659.

Flannery, J., and S. Fisher (1984) Circadian disc shedding in Xenopus retina in vitro. Invest. Ophthalomol. Vis. Sci. 25: 229-232.

Fleissner, G., and G. Fleissner (1978) The optic nerve mediates the circadian pigment migration in the median eyes of the scorpion. Comp. Biochem. Physiol. (A) 61: 69-71.

Fowlkes, D. H., C. J. Karwoski, and L. M. Proenza (1985) Effects of unilateral and bilateral optic nerve transection (ONX) on the anolis ERG circadian rhythm (CR). Invest. Ophthalmol. Vis. Sci. (Suppl.) 26: 112.

Goldman, A., P. Teirstein, and P. O'Brien (1980) The role of ambient lighting in circadian disc shedding in the rod outer segment of the rat retina. Invest. Ophthalmol. Vis. Sci. 19: 1257-1267.

Jacklet, J. (1969) Circadian rhythm of optic nerve impulses recorded in darkness from the isolated eye of Aplysia. Science 164: 562-564.

Kaplan, E., and R. B. Barlow, Jr. (1980) Circadian clock in Limulus brain increases response and decreases noise of retinal photoreceptors. Nature 286: 393-395.

Kass, L., and R. B. Barlow, Jr. (1984) Efferent neurotransmission of circadian rhythms in the Limulus lateral eye. I. Octopamine-induced increases in retinal sensitivity. J. Neurosci. 4: 908-917.

LaVail, M. M., and P. A. Ward (1978) Studies on the hormonal control of circadian outer segment disc shedding in the rat retina. Invest. Ophthalmol. Vis. Sci. 17: 1189-1193.

Lehman, H., and R. B. Barlow, Jr. (1986) Efferent neurotransmitters in the eye of Limulus: Evidence for a neuropeptide. Soc. Neurosci. Abstr. 12: 242.

Levinson, G., and B. Burnside (1981) Circadian rhythms in teleost retinomotor movements. Invest. Ophthalmol. Vis. Sci. 20: 294-303.

Pittendrigh, C. S. (1981) Circadian systems: Entrainment. In Handbook of Behavioral Neurobiology, S. J. Aschoff, ed., pp. 95-123, Plenum, New York.

Renninger, G. H., E. Kaplan, and R. B. Barlow, Jr. (1984) A circadian clock increases the gain of photoreceptor cells of the Limulus lateral eye. Biol. Bull. 167: 532.

Robertson, L. M., and J. S. Takahashi (1985) Circadian clock in cell culture: Oscillation of melatonin release from perfused chick pineal cells. Soc. Neurosci. Abstr. 11: 818.

Takahashi, J. S., H. Hamm, and M. Menaker (1980) Circadian rhythms of melatonin release from individual superfused chicken pineal glands in vitro. Proc. Natl. Acad. Sci. USA 77: 2319-2322.

Teirstein, P. S., A. I. Goldman, and P. J. O'Brien (1980) Evidence for both local and central regulation of rat outer segment disc shedding. Invest. Ophthalmol. Vis. Sci. 19: 1268-1273.

Terman, M., and J. Terman (1985) A circadian pacemaker for visual sensitivity? Ann. NY Acad. Sci. 453: 147-161.

Trump, B. F., E. A. Smuckler, and E. P. Benditt (1961) A method for staining epoxy sections for light microscopy. J. Ultrastruct. Res. 5: 343-348.

Wirz-Justin, A., M. DaPrada, and C. Reme (1984) Circadian rhythms in rat retinal dopamine. Neurosci. Lett. 45: 21-25. 